\title{
Forest before trees? It depends where you look
}

\author{
G. ROBERT GRICE, LYN CANHAM, and JOSEPH M. BOROUGHS \\ University of New Mexico, Albuquerque, New Mexico
}

\begin{abstract}
Because it may be deduced from the more elementary principles of visual processing, global precedence (Navon, 1977) is not a primary perceptual principle. Subjects were presented with a large letter made out of small ones and asked to make an identification response on the basis of either the large or small letter. When fixation was controlled to provide adequate stimulation from the small letter, there was no difference in reaction time (RT) between the large and small targets. Also, there was no difference in interference due to response incompatibility of the unattended letter based on target size. However, when the stimulus was presented peripherally, unpredictably to the right or left of fixation, RT was faster to the large target and interference was substantially greater for the small target. Functions for the development of associative strength and associative interference are presented. Global precedence is dependent on factors tending to degrade small stimuli more than large ones.
\end{abstract}

In reaction time $(\mathrm{R} T)$ experiments of letter identification, the presence in the display of a noise letter associated with a competing response increases RT relative to control conditions for which this is not the case (B. A. Eriksen \& C. W. Eriksen, 1974). A number of variables influencing the effect have been investigated, and C. W. Eriksen and Schultz (1979) have reported such experiments in support of their continuous flow conception of visual processing. Recently, Grice, Canham, and Schafer (1982) investigated the temporal dynamics of the process and identified it as an important example of associative interference.

While this form of interference is extremely robust, there is one interesting case in which it has not been obtained. Navon (1977) utilized a display consisting of a large letter made up of smaller letters, which could be either the same as or different from the large letter they formed. Subjects were asked to respond on the basis of either the large letter as the target or the small letter. With the small target, the typical interference effect was obtained. When the large letter was response-incompatible with the small target, RT was slower than when the large letter was the same. However, when the large letter was the target, response compatibility of the small letter had no effect. In addition, RTs were faster to the large target than to the small target. Navon suggested that these findings support a principle of global precedence, meaning that "perception proceeds from global

This research was supported by PHS Grant MH 16400 from the National lnstitute of Mental Health. Requests for reprints should be sent to G. Robert Grice, Department of Psychology, University of New Mexico, Albuquerque, New Mexico 87131. analysis to more and more fine-grained analysis" (Navon, 1977, p. 381).

Retrospectively, it is not very surprising that Navon obtained the results he did concerning the interference effect. Stimuli were presented unpredictably in one of the four quadrants of the display with respect to a central fixation point. The exposure was brief $(40 \mathrm{msec})$, and the stimulus was followed immediately by a mask. C. W. Eriksen and Schultz (1979) found that the size of the compatibility effect was affected by both the relative size and relative distinctiveness of target and noise letters. All of Navon's procedures would be expected to degrade small stimuli more than large ones, a state which would lead to the obtained results.

More recent research has indicated that global precedence is less than ubiquitous. Kinchla and Wolfe (1979), using stimuli similar to Navon's, presented the display in several sizes. Subjects were asked to respond yes or no with respect to the presence of a target letter. As long as the large letter was less than $8 \mathrm{deg}$, they responded faster if the target was the large letter, a result consistent with global precedence. However, at sizes greater than $8 \mathrm{deg}$, they responded faster to the small target. The findings confirmed the authors' initial hypothesis that "shapes which are very large must project identifying contours on lowresolution regions of the retina, while the constituent lower-order forms fall on high-acuity regions near the fovea"' (Kinchla \& Wolfe, 1979, p. 226). The question remains, however, as to the extent that this size relation would hold with the uncertain location procedure used by Navon.

Martin (1979) found global precedence when the large letter was formed by small letters in a $5 \times 7$ matrix but not when the matrix was $3 \times 5$. Hoffman 
(1980) failed to find the effect when the large letter was presented in distorted form. Of course, these experiments may be answered by the reply that there would be no reason to expect global precedence if the large form did not look very much like the letter that it was supposed to represent. In any case, the above evidence does indicate that global precedence does have boundary conditions and is not an entirely general phenomenon.

The intent of the present research was to examine further the generality of the principle of global precedence with the same class of stimuli used by Navon, but in a situation favorable for clear perception of the small letters. The letter of the size that the subject is instructed to ignore is regarded as noise. The research consists of four experiments comparing the effects of same-letter, irrelevant, and responseincompatible noise. Two experiments, one with the large target and one with the small, were conducted with the stimulus in a fixed location, and with fixation in the center of the large letter. In the other two experiments, the stimuli were presented peripherally to the right or left of the fixation point, in an unpredictable order. The target letters were $\mathrm{H}$ and $\mathrm{S}$, those used by Navon. The interference effect was evaluated by comparing the same-letter and incompatible-noise conditions. We also used the methods of variable criterion theory to investigate the development of associative strength and associative interference in these conditions. The irrelevant-noise condition was merely exploratory, to see what would happen with an irrelevant letter bearing some featural similarity to both of the targets. For this we used the letter $B$.

\section{METHOD}

\section{Subjects}

The subjects were 112 undergraduate women from courses in introductory psychology. They received course credit for participation. There were 28 subjects in each of the four experiments.

\footnotetext{
Apparatus and Stimuli

The subjects were seated in a sound-attenuating chamber before a small table and in front of a TV monitor on which the stimuli were presented. Two telegraph keys on the table were operated by the two index fingers. The stimuli were controlled and the timing accomplished by a TRS-80, Model I microcomputer (Grice, 1981). Stimuli were large capital letters made up of small letters. The small letters were the regular TRS-80 double-width capital letters which are formed by a $5 \times 7$ dot matrix. They were light on a dark background. The large letters were also formed in a $5 \times 7$ matrix with a space between the vertical columns to create approximately equal vertical and horizontal spacing. The large target letters, $\mathrm{H}$ and $\mathrm{S}$, were formed by filling the same cells with small letters as were filled with dots in the small letters. The large letter used as irrelevant noise, B, differed from the small B only in that the straight vertical line was placed in the extreme left column rather than in the second column. This was to make it more equally similar to $\mathrm{H}$ and $\mathrm{S}$.
}

The viewing distance to the screen was about $114 \mathrm{~cm}$. At this distance, the small letters were $.29 \mathrm{deg}$ wide and $.35 \mathrm{deg}$ high. The large letters were $2.76 \mathrm{deg}$ wide and $3.57 \mathrm{deg}$ high. A plus sign was used as a fixation point and appeared in the center of the screen at all times except when the stimuli were on. For experiments with fixed stimulus location, this point was in the center of the large letter. For experiments with uncertain stimulus location, the large letter appeared with its center $2.47 \mathrm{deg}$ either to the left or right of the center of fixation. The distance from the fixation point to the interior edge of the large letter was $1.09 \mathrm{deg}$. The large letter required $5 \mathrm{msec}$ to be printed from top to bottom, and timing of the RT, in milliseconds, began with its completion. The fixation point went off as the stimulus came on.

\section{Procedure}

The subjects were instructed to respond to $\mathrm{H}$ with the right, or left, key and to $S$ with the other key. Half of the subjects received each mapping, but the variable produced no significant effects and is disregarded in the subsequent analyses. Instructions were to respond as quickly as possible while avoiding errors. A warning signal before each trial consisted of a $.5-\mathrm{sec}, 1,000-\mathrm{Hz}$ tone of $70 \mathrm{~dB}$ SPL. The interval from the onset of the warning signal to stimulus onset was $1.0,1.2,1.5,1.8$, or $2.0 \mathrm{sec}$ equally often in an irregular order. The subjects were told to look at the fixation point at this signal and to be prepared to respond. Stimuli remained on until the subject responded. The experiment began with 50 practice trials followed by a short rest. At this point, any questions were answered or any necessary reinstruction given. There followed 250 trials without interruption. The first 10 of these were treated as further practice. Data are based on the final 240 trials, 80 with each of the three noise types-same letter, irrelevant, and incompatible, in an irregular order. When an error occurred, the word "ERROR" appeared on the screen for $.5 \mathrm{sec}$ and a .5-sec burst of noise, $70 \mathrm{~dB}$ SPL, sounded in the earphones. The interval between trials was 4 sec.

Fixed location. In these two experiments, the large letter always appeared in the middle of the screen with the location of the fixation point at the center. In one experiment, the subjects were instructed to respond to the large letter and ignore the small ones. The large letter was made up of small $\mathrm{Hs}$, Bs, or Ss, depending upon the noise type, and the large letter was either an $H$ or an $S$, each half of the time for each noise type. In the second experiment, the subjects were instructed to respond to the small letter and ignore the large letter. Here the small letters were either $H$ or $S$, and the large letter was $\mathrm{H}, \mathrm{B}$, or $\mathrm{S}$.

Uncertain location. These two experiments were the same as above, except that the large letter appeared either to the right or left of the fixation point in an irregular order. The frequencies of right and left presentations were equated for each target and for each noise condition.

\section{RESULTS}

Mean RTs and error rates are presented in Table 1. Error rates are generally low, with few significant differences. With the large-target and fixed-stimulus location, the error rate for incompatible noise is significantly greater than for same noise. According to the sign test, $\mathrm{p}<.01$. With the small target and uncertain position, errors for incompatible noise are significantly greater than for both same and irrelevant noise ( $p<.001$ in both instances). No other differences in error rate are significant. Mean RTs are fastest for same noise and slowest for incompatible noise in all experiments. Our primary interest here is the comparison of these two conditions across the different experiments. 
Table 1

Means and Standard Deviations of Mean Correct Reaction Time (in Milliseconds) and of Error Rates

\begin{tabular}{|c|c|c|c|c|c|c|c|c|c|c|c|c|}
\hline \multirow{4}{*}{$\begin{array}{l}\text { Tar- } \\
\text { get }\end{array}$} & \multicolumn{12}{|c|}{ Noise Condition } \\
\hline & \multicolumn{6}{|c|}{ Reaction Time } & \multicolumn{6}{|c|}{ Error Rate } \\
\hline & \multicolumn{2}{|c|}{ Same } & \multicolumn{2}{|c|}{ Irrelevant } & \multicolumn{2}{|c|}{ Incompatible } & \multicolumn{2}{|c|}{ Same } & \multicolumn{2}{|c|}{ Irrelevant } & \multicolumn{2}{|c|}{ Incompatible } \\
\hline & Mean & SD & Mean & $\mathrm{SD}$ & Mean & SD & Mean & $\mathrm{SD}$ & Mean & $\mathrm{SD}$ & Mean & SD \\
\hline & \multicolumn{12}{|c|}{ Jixed Location Experiment } \\
\hline \multirow[t]{2}{*}{ Small } & $\begin{array}{l}452.2 \\
454.5\end{array}$ & $\begin{array}{l}65.7 \\
61.3\end{array}$ & $\begin{array}{l}470.8 \\
488.0\end{array}$ & $\begin{array}{l}69.6 \\
74.9\end{array}$ & $\begin{array}{l}489.1 \\
491.1\end{array}$ & $\begin{array}{l}77.1 \\
71.0\end{array}$ & $\begin{array}{l}.010 \\
.011\end{array}$ & $\begin{array}{l}.016 \\
.016\end{array}$ & $\begin{array}{l}.012 \\
.016\end{array}$ & $\begin{array}{l}.014 \\
.018\end{array}$ & $\begin{array}{l}.026 \\
.020\end{array}$ & $\begin{array}{r}.029 \\
.020\end{array}$ \\
\hline & \multicolumn{12}{|c|}{ Uncertain Location Experiment } \\
\hline $\begin{array}{l}\text { Large } \\
\text { Small }\end{array}$ & $\begin{array}{l}455.0 \\
510.6\end{array}$ & $\begin{array}{l}62.6 \\
64.1\end{array}$ & $\begin{array}{l}459.2 \\
571.8\end{array}$ & $\begin{array}{l}63.2 \\
64.9\end{array}$ & $\begin{array}{l}466.8 \\
592.7\end{array}$ & $\begin{array}{l}66.1 \\
72.7\end{array}$ & $\begin{array}{l}.016 \\
.009\end{array}$ & $\begin{array}{l}.016 \\
.012\end{array}$ & $\begin{array}{l}.018 \\
.015\end{array}$ & $\begin{array}{l}.015 \\
.023\end{array}$ & $\begin{array}{l}.012 \\
.046\end{array}$ & $\begin{array}{l}.013 \\
.043\end{array}$ \\
\hline
\end{tabular}

\section{Fixed Stimulus Location}

With the fixed stimulus location, RTs to the large and small targets were about the same and do not differ significantly $[F(1,54)<1]$. The associative interference effect clearly appeared in the difference between same and incompatible noise and is significant $[F(1,54)=88.34, p<.001]$. Also, the difference is almost exactly the same for the large and small targets. The interaction of noise type $x$ target size is not significant $[F(1,54)<1]$. Evidence for global precedence would have included faster RT to the large target and a substantially greater interference effect with the small target. Clearly, there is no such evidence in these data.

\section{Uncertain Stimulus Location}

Results were quite different with the uncertain stimulus location, and provide a clear demonstration of the global precedence effect. RTs were significantly faster to the larger target $[F(1,54)=27.04, p<.001]$. The interference effect in this condition is substantially greater for the small target, and the noise type $x$ target size interaction is significant $[F(1,54)=$ $108.61, p<.001$ ]. Navon (1977) found no interference effect for the large target, and in this one respect our data differ from his. Although small, the difference between incompatible and same noise is significant $[\mathrm{t}(27)=3.107, \mathrm{p}<.01]$. Possibly a brief exposure and masking would have eliminated this effect.

The entire pattern of results is presented graphically in Figure 1, where mean RTs for same and incompatible noise are shown for each target size and each location condition. An analysis of all experiments verifies the different conclusions of the separate analyses presented above. The effect of target size on RT does, in fact, depend upon stimulus location. The interaction of target size and location is significant $[F(1,108)=12.46, p<.001]$. Furthermore, the dependency of the compatibility effect on target size varies significantly with stimulus location. For the three-way interaction, noise type $\times$ target size $\times$ location, $F(1,108)=46.84, p<.001$.
In the presence of a three-way interaction, it may be instructive to view the data from more than one perspective. The separate $2 \times 2$ analyses presented above were for each location condition. Alternatively, we have computed separate analyses for each target size with noise type and stimulus location as the variables. For the large target, the compatibility effect was significant $[F(1,54)=58.82, p<.001]$. The effect of location was not $[F(1,54)<1]$. The interaction, location $\times$ compatibility, was significant $[F(1,54)=15.69, p<.001]$. For the small target, compatibility was significant $[F(1,54)=211.95, p<$ $.001]$. In this case, the primary effect of location was also significant $[F(1,54)=20.14, p<.001]$. The compatibility $\times$ location interaction was again significant $[F(1,54)=31.23, p<.001]$. The interesting point of the two significant interactions is that they are in opposite directions. For the large target, the effect of location uncertainty was to decrease the compatibility effect, but for the small target, the result was a substantial increase. This aids in under-

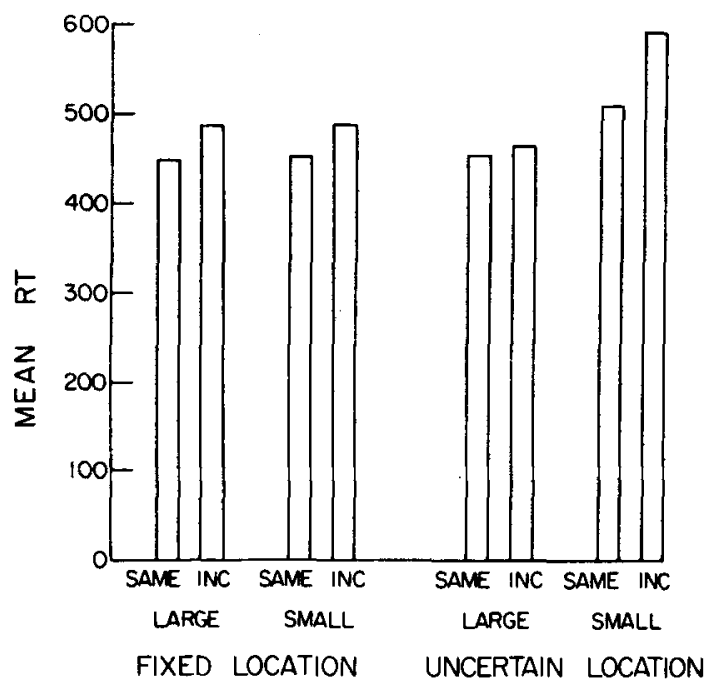

Figure 1. Mean reaction time in milliseconds for same letter and incompatible noise in each experiment. 
standing the powerful triple interaction, and leads to a rather straightforward interpretation of the findings. Uncertainty of stimulus location degrades the small letter more than the large one. Thus, if the small letter is noise, its interference effect with the large target is decreased by the uncertain location. However, if the small letter is the target, the result is an increase in the interference effect of the large letter.

\section{Growth of Associative Strength}

The principle of global precedence suggests that global information accumulates earlier than local information. For this reason, we have applied the scaling methods of variable criterion theory (Grice, Nullmeyer, \& Spiker, 1982; Grice, Canham, \& Schafer, 1982) in order to examine the details of this possibility. The analysis was conducted with the cumulative group RT distribution for same and incompatible noise in each experiment. The race model of Grice, Spiker, and Nullmeyer (1979) was applied to the proportions of correct responses and errors to obtain functions indicating the theoretical probability that the correct response had reached criterion by successive times. ${ }^{1}$ The shapes of functions obtained in this way are invariant with respect to the number of errors. Functions indicating the growth of excitatory strength in scale units consist of the normal deviates of the theoretical proportions. The model used here is similar to the one recently used by Grice, Canham, and Shafer (1982) for letter identification. For the same-noise condition, the relation is

$$
E(t)=[A(t)-\bar{C}] / o,
$$

where $E(t)$ is the excitatory strength of the correct response at time, $t$, following stimulus onset, and $A(t)$ is the amount of associative strength relating the target stimulus to the response. $\overline{\mathrm{C}}$ and $\sigma$ are the mean and standard deviation of the criterion distributions. For the incompatible-noise condition, the model is

$$
E(t)=[A(t)-A I(t)-\bar{C}] / o,
$$

where $\mathrm{Al}(\mathrm{t})$ is the amount of associative interference at time $t$. Since the analysis of each experiment is on the scale of that experiment, the values of $\bar{C}$ and $\sigma$ are always 0 and 1 , and do not appear in any of the equations.

For the same-letter noise conditions in each of the four experiments, the functions for the growth of associative strength were smooth, negatively accelerated functions which were very well fitted by exponential growth functions. This is the same as for the identical-noise conditions in the flanker task studied by Grice, Canham, and Schafer (1982). These functions are presented graphically in Figure 2. All fitted equations are presented in the Appendix. In the fixedlocation experiments, the functions for the large and small targets are about the same, although the large target function is slightly above that for the small target at the earliest intervals. However, this difference is small and the functions are essentially superimposable through most of their range. If the two obtained functions are plotted against each other at corresponding intervals, they share $99.6 \%$ common variance with the relation $y=x$. Of course, no difference in mean RT was produced. The global precedence effect is clearly evident for the uncertain location, since the function for the small target is delayed relative to the large target throughout the entire range. There was no trace in the data of an inflected form for the small target function-a possibility which could be inferred from a suggestion of Navon (1981a).

On the basis of Equation 2, functions for associative interference (AI) were initially estimated by subtracting the obtained values of $E(t)$ from the calculated functions for $A(t)$ in each experiment. It was at once apparent that the functions for the large and small targets were not of the same shape. For the large target with small noise letters, AI increased monotonically. However, for the small target with large noise letters, the functions first increased, reached a maximum, and then decreased. This was the same kind of function found by Grice et al. (1982) for
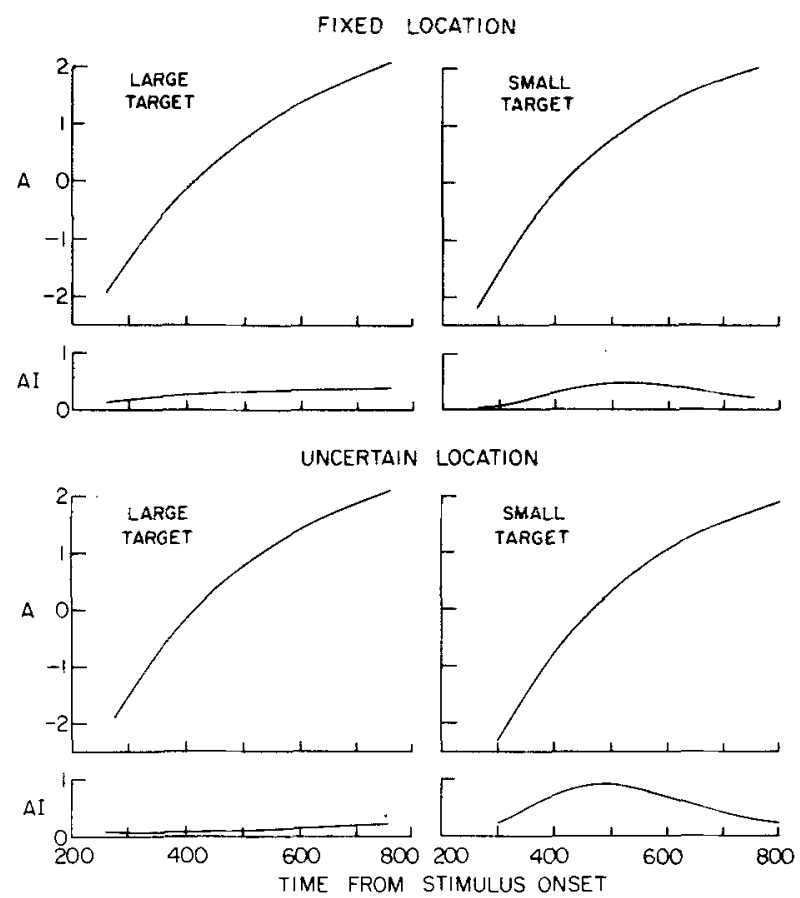

Figure 2. Graphic representation of the theoretical functions for associative strength (A) and associative interference (AI) for each experiment. Time from stimulus onset is in milliseconds. 
target and incompatible noise letters of the same size in the flanker task. As in that analysis, these were fitted by differential equations based on the Gompertz growth function. In the case of the large target, it was preferable to describe the $E$ values for incompatible noise by exponential growth functions. Calculated functions for $\mathrm{AI}(\mathrm{t})$ were the differences between these functions and those of $A(t)$.

The four functions for the development of associative interference are also presented in Figure 2. For the fixed location, interference produced by the large noise letters shows faster growth once underway, but at short latencies it is less than that produced by the small noise letter. In other words, what is demonstrated here is local, not global, precedence. The net result was that total interference was the same for the large and small targets. For the uncertain location, interference in the small-target experiment is above that for the large target not only early but throughout. Actually, it is not merely global precedence, but a greater continued interference effect as well. It should also be noted that the large noise letter has an earlier and greater interference effect with uncertain location than it does with fixed location.

The nonmonotonic effect of associative interference for the large noise letters agrees with the findings of Grice et al. (1982) in five experiments with flanker noise letters of the same size as the target. They suggested the tentative interpretation that, if the correct and competing responses tend to approach criterion about the same time, the incorrect response may be actively inhibited. This inhibition may also delay the correct response. If the inhibition is successful, the delayed correct responses will occur later, accounting for the recovery phase.

The obtained and calculated cumulative group RT distributions for same and incompatible noise in each experiment are presented in Figure 3. Calculations were made on the basis of Equations 1 and 2, the fitted equations, and the predictive race model of Grice et al. (1979). What this analysis shows is that the set of orderly functions obtained by applying the measurement operations of variable criterion theory does a very good job of describing the data. The proportion of variance accounted for by the calculated values is .999 or higher for each of the eight obtained distributions.

\section{Effects of the Irrelevant Noise Letter}

The exploratory condition employing a noise letter bearing some featural similarity to each target produced mean RTs intermediate between same and incompatible noise in all experiments. For fixed position with the large target, it differed significantly from both same noise $[\mathrm{t}(27)=4.960, \mathrm{p}<.001]$ and incompatible noise $[\mathrm{t}(27)=3.71, \mathrm{p}<.001]$. For fixed position, small target, it differed significantly only from

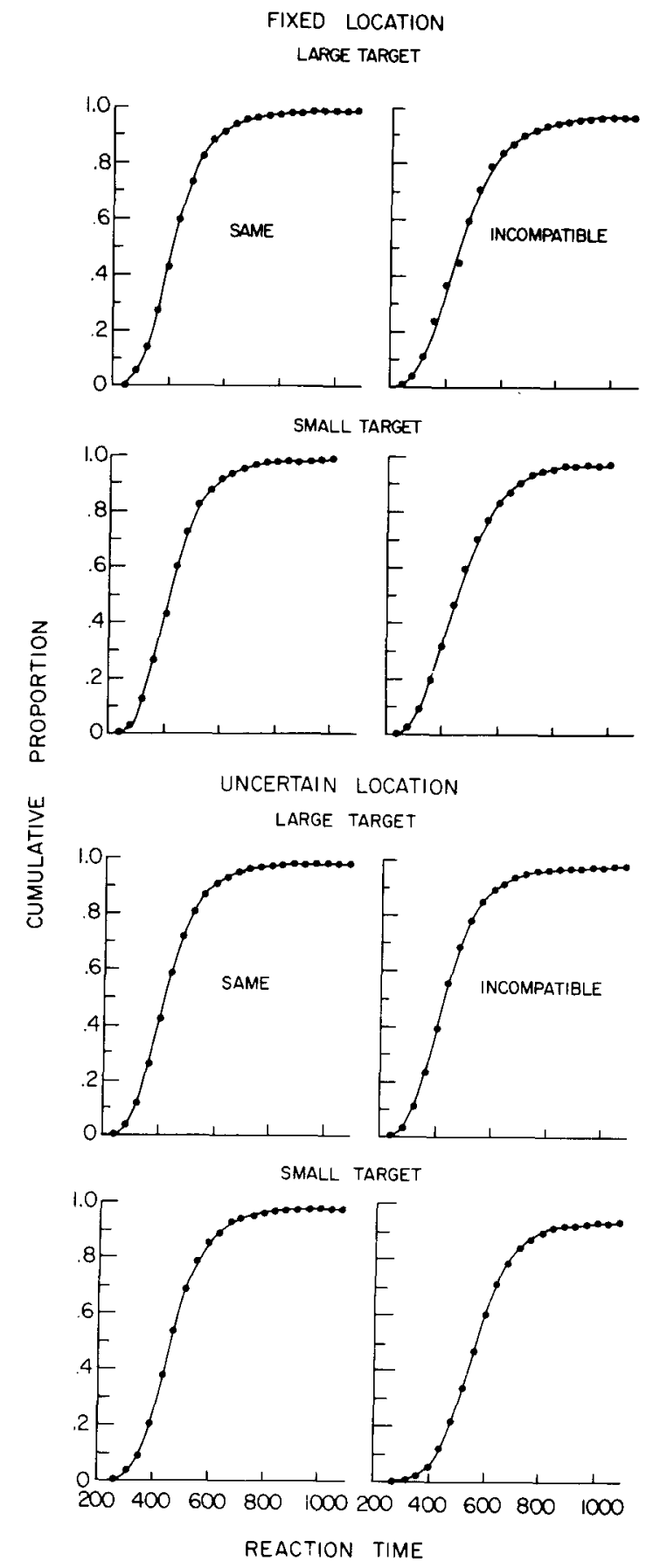

Figure 3. Calculated and obtained cumulative RT distributions for same-letter and incompatible noise for each experiment. RT is in milliseconds.

same noise $[\mathrm{t}(27)=5.906, \mathrm{p}<.001]$. While irrelevantnoise RTs were slower for the small target than for the large one, the difference was not significant [t(54) $=.889$, n.s.]. For uncertain position with the large target, irrelevant noise differed significantly only 
from incompatible noise $[\mathrm{t}(27)=2.417, \mathrm{p}<.01]$. With the small target and uncertain position, it differed significantly from both same noise $[\mathrm{t}(27)=11.456$, $\mathrm{p}<.001]$ and incompatible noise $[\mathrm{t}(27)=3.663$, $\mathrm{p}<.001]$. When compared with same-letter noise over all experiments, the pattern is essentially similar to that of incompatible noise, but the effects are smaller. The triple interaction of noise type $\times$ target size $\times$ location is significant $[\mathrm{F}(1,108)=22.05, \mathrm{p}<$ $.001]$. It is suggested that this condition also produced associative interference.

\section{DISCUSSION}

Global precedence is not a universal perceptual principle. When fixation is controlled to insure adequate reception of the local stimuli, no such evidence is obtained. RTs to the large and small targets are the same, and equal interference is produced by large and small letters acting as noise. On the other hand, the global precedence effect may be produced with the same stimuli if they are presented peripherally to the right or left of fixation in an unpredictable fashion. Presumably, any factor tending to degrade small stimuli more than larger ones would tend to produce the effect. This would include such variables as uncertainty of location, location stimulated on the retina, exposure duration, masking, and clarity. This conclusion is similar to that reached by Hoffman (1980). It is also in essential agreement with more recent statements of Navon (1981a, 1981b). In these more recent statements, he has suggested that global precedence has considerable ecological validity in that things are frequently viewed in such a way as to produce it. This suggestion undoubtedly has merit. However, it should be recognized that global precedence is not a primary principle but a set of phenomena deducible from more elementary principles of visual processing.

The analysis in terms of variable criterion theory appears to have contributed materially to the understanding of the situation. With fixed stimulus location, favorable to perception of the small letters, the growth of associative strength is about the same for the large and small targets. However, with uncertain stimulus location, less favorable for perception of the small letters, associative strength shows considerably earlier growth for the large target. Comparison of the associative functions for each target size across conditions are also of interest, that is, the vertical comparisons in Figure 2. For the large letter, location has very little effect and the two functions are about the same. However, for the small letter, the growth of associative strength was considerably delayed by uncertainty of location. It is this comparison, in particular, that illustrates the differentially degrading effect of uncertain location on the small target.
Results of the analysis of the development of associative interference are a little more complex. For the uncertain location, it is clear enough that interference by the large letter with the small target is both earlier and much greater than for the reverse arrangement. With so little interference in the large-target condition, the function is necessarily quite flat and its exact form should probably not be taken very seriously. In the fixed-location experiments, the two functions indicate about the same amount of interference, but they have different forms. The function for interference by the small letter on the large target began at a higher level than did that for the small target. It then increased very gradually. This actually indicates a form of local precedence in interference. Presumably, it reflects the fact that the subject initially fixated the position of a small letter. For the same reason, interference by the large noise letter began at a lower level, but then increased at a more rapid rate. This delayed, but more rapid, growth of interference possibly reflects a greater salience of the large letter as fixation continues. The question remains as to why the function for the large target was monotonic but that for the small target was not. In accord with our hypothesis that the interference is based on the inhibition of errors, the fairly abrupt rise of the function for the small target suggests that there were numerous delayed responses within a relatively short time of the distribution. These delayed responses would then tend to occur within a similar, later period, producing the recovery phase of the nonmonotonic function. With the much more gradual function for the large target, the delayed responses were more widely distributed, and their recovery merely blended with the regular growth of associative strength.

Boer and Keus (1982), who favor a stage interpretation, have suggested that global precedence, or dominance, is a postperceptual effect. Their argument was based on the finding that there was no difference between same letter and incompatible conditions in the intercepts of fitted, linear speed-accuracy tradeoff functions. However, the intercepts of such functions involve considerable extrapolation beyond the data, and reasoning on this basis is extremely tenuous. From our own data, a tradeoff function may be obtained for the uncertain-location, smalltarget experiment as the difference in excitatory strength between correct responses and errors. This was called a variable criterion discrimination function by Grice and Spiker (1979). This function for these data is sigmoid in form, with initial positive acceleration, and a linear fit would be inappropriate. In any case, our analysis clearly shows that the global precedence effect with uncertain location is present from the earliest responses, as indicated by RTs to the large and small targets and by the asymmetrical interference effect. 
In a recent exchange between Miller (1981) and Navon (1981b), Miller suggested an attentional interpretation of global precedence, while Navon favored a perceptual explanation. Our results with manipulation of stimulus location in relation to fixation tend to support the perceptual interpretation of the effect when it occurs. This is in accord with previous findings that the effect' may be manipulated by other perceptual variables, for example, stimulus size (Kinchla \& Wolf, 1979) and stimulus distortion (Hoffman, 1980). Miller presented evidence from RT distributions that local and global information were present simultaneously. This agrees with our own findings. However, neither Navon nor we (1981b) believe that this demands an explanation in terms of attention. All that is required to obtain the effect on a perceptual basis is that the two sources of information grow at different rates.

It should be mentioned that Miller (1981) did obtain evidence for global precedence with a fixed stimulus location and a central fixation point. His experiment differed from ours in numerous respects. However, the most relevant difference is probably the manner in which fixation was controlled. In his experiment, the screen was blank between trials and the only indication that a trial was about to begin was the appearance of the fixation point for only $250 \mathrm{msec}$. It was then off for another $250 \mathrm{msec}$ before the stimulus appeared. In the present experiments, the fixation point was on at all times except during stimulus presentation. At the auditory warning signal, the subject had an average of $1.5 \mathrm{sec}$ to fixate and remain fixated until the stimulus appeared. This procedure obviously provided better control of fixation, and probably is the major factor accounting for the different findings. Hoffman (1980), also using a centrally fixated display, found no evidence of global precedence when stimuli of this kind were used as probe stimuli in an item-recognition task.

Both Miller (1981) and Navon $(1977,1981 \mathrm{~b})$ have suggested a possible basis for an asymmetrical interference in this situation. It was suggested that subjects may be able successfully to attenuate or filter sensory input from the small letter but not from the large one. However, no such effect was present in our fixed-position condition. With fixation appropriately controlled, subjects responding to the large target were just as unable to avoid processing the small noise letters as is true in the flanker task with noise and target of the same size.

Since we believe in a continuous-growth interpretation of information processing and response evocation rather than discrete stage theory, we cannot take seriously the logic of the method of additive factors (Sternberg, 1969). However, in view of widespread interest in this logic, we should emphasize certain aspects of the present data. Stage theorists would generally regard target size and certainty of location as affecting an early perceptual or encoding stage, while response compatibility should affect a later response-selection stage. Yet there are significant interactions of compatibility with both of the other variables. Additive-factors logic states that variables affecting different stages should have additive effects and not interact. If this logic is valid with respect to stage theory, these results provide obvious difficulty. The interactions raise no difficulty for a continuousgrowth interpretation.

We have consistently referred to the difference between same letter and response-incompatible noise as interference. This is based on a much larger body of evidence that such arrangements do produce it. We recognize, however, that some of the effect may be a facilitative effect of the same letter. Our research does not answer that question. The significant differences between incompatible and irrelevant noise at least indicate that facilitation by the same letter is not the entire effect.

\section{REFERENCES}

Boer, L. C., \& Kuess, P. J. G. Global precedence as a postperceptual effect: An analysis of speed-accuracy tradeoff functions. Perception \& Psychophysics, 1982, 31, 358-366.

Eariksen, B. A., \& ERIKsen, C. W. Effects of noise letters upon the identification of a target letter in a nonsearch task. Perception \& Psychophysics, 1974, 16, 143-149.

ERIKSEN, C. W., \& SCHULTZ, D. W. Information processing in visual search: A continuous flow conception and experimental results. Perception \& Psychophysics, 1979, 25, 249-263.

GRICE, G. R. Accurate reaction time research with the TRS-80 microcomputer. Behavior Research Methods \& Instrumentation, 1981, 13, 674-676.

Grice, G. R., Canham, L., \& Schafer, C. Development of associative and perceptual interference. Perception \& Psychophysics, 1982, 32, 375-387.

Grice, G. R., Nullmeyer, R., \& Spiker, V. A. Human reaction time: Toward a general theory. Journal of Experimental Psychology: General, 1982, 111, 135-153.

Grice, G. R., \& Spiker, V. A. Speed-accuracy tradeoff in choice reaction time: Within conditions, between conditions, and between subjects. Perception \& Psychophysics, 1979, 26, 118-126.

Grice, G. R., SpIker, V. A., \& Nullme Yer, R. Variable criterion analysis of individual differences and stimulus similarity in choice reaction time. Perception \& Psychophysics, 1979, 25, 353-370.

Hoffman, J. E. Interaction between global and local levels of a form. Journal of Experimental Psychology: Human Perception and Performance, 1980, 6, 222-234.

Kinchla, R. A., \& Wolfe, J. M. The order of visual processing: "Top-down," "bottom-up," or "middle-out." Perception \& Psychophysics, 1979, 25, 225-231.

Martin, M. Local and global processing: The role of sparsity. Memory \& Cognition, 1979, 7, 476-484.

MiLler, J. Global precedence in attention and decision. Journal of Experimental Psychology: Human Perception and Performance, 1981, 7, 1161-1174.

Navon, D. Forest before trees: The precedence of global features in visual perception. Cognitive Psychology, 1977, 9, 353-383.

Navon, D. The forest revisited: More on global precedence. Psychological Research, 1981, 43, 1-32. (a) 
Navon, D. Do attention and decision follow perception? Comment on Miller. Journal of Experimental Psychology: Human Perception and Performance, 1981, 7, 1175-1182. (b)

Sternbero, S. The discovery of processing stages: Extensions of Donders' method. Acta Psychologica, 1969, 30, 276-315.

\section{NOTE}

1. A brief summary of the scaling procedures and the race model used in variable criterion theory has recently been presented in an Appendix by Grice et al., 1982.

\section{APPENDIX}

The exponential growth functions for associative strength for same letter noise are as follows:

Fixed position, large target:

$$
A(t)=3.223-11.455 e^{-.00306 t}
$$

Fixed position, small target:

$$
A(t)=2.779-13.527 e^{-.00382 t}
$$

Uncertain position, large target:

$$
A(t)=2.956-12.839 \mathrm{e}^{-.00353 \mathrm{t}} \text {. }
$$

Uncertain position, small target:

$$
A(t)=2.651-15.703 \mathrm{e}^{-.00380 t} \text {. }
$$

The exponential growth functions for excitatory strength for the incompatible noise, larger targets are as follows:

Fixed position:

$$
E(t)=2.800-10.623 \mathrm{e}^{-.003001} \text {. }
$$

Uncertain position:

$$
E(t)=2.647-12.966 \mathrm{e}^{-.00375 t}
$$

In obtaining functions for associative interference for the small targets, incompatible-noise Gompertz growth functions were first fitted to the estimates of $\mathrm{AI}(\mathrm{t})$ summated at 20-msec intervals. These functions are as follows:

Fixed position:

$$
\Sigma \mathrm{Al}(\mathrm{t})=9.5\left(6.568 \times 10^{-14}\right) .9935^{\mathrm{t}}
$$

Uncertain position:

$$
\Sigma A I(t)=17.5\left(8.094 \times 10^{-14}\right)^{.993^{t}} .
$$

The Gompertz function may be written as

$$
\Sigma A I(t)=v g^{h^{t}} .
$$

The differential equations describing $\mathrm{AI}(\mathrm{t})$ are then computed at 20-msec intervals by the following expression:

$$
A l(t)=v g^{h t} h^{t}(\ln g)(\ln h) \times 20 .
$$

(Manuscript received July 12, 1982; revision accepted for publication October 22, 1982.) 